\title{
Differential Regulation of Active Zone Density during Long-Term Strengthening of Drosophila Neuromuscular Junctions
}

\author{
Dierk F. Reiff, Philippe R. Thiel, and Christoph M. Schuster \\ Friedrich-Miescher-Laboratorium der Max-Planck-Gesellschaft, 72076 Tübingen, Germany
}

In this study we established a transgenic $\mathrm{Ca}^{2+}$ imaging technique in Drosophila that enabled us to target the $\mathrm{Ca}^{2+}$ sensor protein yellow Cameleon-2 specifically to larval neurons. This noninvasive method allowed us to measure evoked $\mathrm{Ca}^{2+}$ signals in presynaptic terminals of larval neuromuscular junctions (NMJs). We combined transgenic $\mathrm{Ca}^{2+}$ imaging with electrophysiological recordings and morphological examinations of larval NMJs to analyze the mechanisms underlying persistently enhanced evoked vesicle release in two independent mutants. We show that persistent strengthening of junctional vesicle release relies on the recruitment of additional active zones, the spacing of which correlated with the evoked presynaptic $\mathrm{Ca}^{2+}$ dynamics of individual presynaptic terminals. Knock-out mutants of the postsynaptic glutamate receptor (GluR) subunit DGluR-IIA, which showed a reduced quantal size, developed NMJs with a smaller number of presynaptic boutons but a strong compensatory increase in the density of active zones. This resulted in an increased evoked vesicle release on single action potentials and larger evoked $\mathrm{Ca}^{2+}$ signals within individual boutons; however, the transmission of higher frequency stimuli was strongly depressed. A second mutant ( $p a b p^{P 970 /+}$ ), which showed enhanced evoked vesicle release triggered by elevated subsynaptic protein synthesis, developed NMJs with an increased number of presynaptic boutons and active zones; however, the density of active zones was maintained at a value typical for wild-type animals. This resulted in wild-type evoked $\mathrm{Ca}^{2+}$ signals but persistently strengthened junctional signal transmission. These data suggest that the consolidation of strengthened signal transmission relies not only on the recruitment of active zones but also on their equal distribution in newly grown boutons.

Key words: transgenic $\mathrm{Ca}^{2+}$ imaging; Cameleon-2; presynaptic $\mathrm{Ca}^{2+}$; long-term strengthening; active zone density; consolidation; synaptic protein synthesis; glutamate receptor; neuromuscular junction; Drosophila
During the rapid growth of developing Drosophila larvae, the body wall muscle cells experience a continuous drop in input resistance (Lnenicka and Keshishian, 2000). To maintain an efficient depolarization of growing muscle cells at all times of development, larval neuromuscular junctions (NMJs) undergo proportional morphological and functional changes that result in enhanced release of presynaptic vesicles and a concomitant increase of excitatory junctional current (EJC) amplitudes. A closely related compensatory mechanism maintains evoked muscle depolarization even in mutants with genetically reduced postsynaptic excitability (Petersen et al., 1997; Davis et al., 1998; DiAntonio et al., 1999). These data have suggested that the level of muscle depolarization is monitored continuously and regulated homeostatically to ensure efficient muscle contraction at all times of larval development (Davis and Goodman, 1998; Paradis et al., 2001).

Besides this developmental control of muscle depolarization, enhanced EJCs (eEJCs) can be further enhanced by the genetic

\footnotetext{
Received July 8, 2002; revised Aug. 15, 2002; accepted Aug. 23, 2002.

This work was funded by the Max-Planck-Society. We thank A. Borst [Department of Neurobiology, Max-Planck-Institute (MPI), Martinsried] for his advice and helpful discussions during early phases of this project. We thank R. Y. Tsien (University of California, San Diego, La Jolla, CA) and C. S. Goodman (University of California at Berkeley, Berkeley, CA) for kindly providing reagents. We thank A. Borst, S. J. Sigrist, W. Hoch (University of Bristol, Bristol, UK) and R. Friedrich (Department of Medical Research, MPI, Heidelberg, Germany) for helpful comments on this manuscript.

Correspondence should be addressed to Christoph M. Schuster, FriedrichMiescher-Laboratorium der Max-Planck-Gesellschaft, Spemannstrasse 39, 72076 Tübingen, Germany. E-mail: christoph.schuster@tuebingen.mpg.de.

D. F. Reiff's current address: Max-Planck-Institute of Neurobiology, Neuronale Informationsverarbeitung, Am Klopferspitz 18a, 82152 Martinsried, Germany. Copyright (C) 2002 Society for Neuroscience $\quad 0270-6474 / 02 / 229399-11 \$ 15.00 / 0$
}

manipulation of neuronal activity and cellular events downstream of neuronal activity, such as increased cellular cAMP levels (Budnik et al., 1990; Schuster et al., 1996; Cheung et al., 1999), elevated CREB- and AP1-dependent transcription (Davis et al., 1996; Sanyal et al., 2002), increased subsynaptic protein synthesis (Sigrist et al., 2000), and altered subunit composition of postsynaptic glutamate receptors (GluRs) (Sigrist et al., 2002). All examples of this adaptive form of junctional strengthening appeared to also rely on an increased evoked release of presynaptic vesicles.

In this study we set out to assess the cellular mechanisms that are involved in the control of persistently strengthened evoked vesicle release at developing NMJs. We first established a new, noninvasive, presynaptic $\mathrm{Ca}^{2+}$ imaging approach in Drosophila, which was based on the targeted transgenic expression of the $\mathrm{Ca}^{2+}$ reporter yellow Cameleon-2 (yCam2) (Miyawaki et al., 1997) in larval neurons and thus in presynaptic terminals of NMJs. We then undertook a detailed analysis of the presynaptic $\mathrm{Ca}^{2+}$ dynamics, junctional signal transmission, and endplate morphology of wild-type and mutant animals that represented both above-mentioned forms of junctional strengthening. These included the knock-out mutant of the postsynaptic glutamate receptor subunit gene $\operatorname{dglurII} A$, which showed strongly reduced quantal sizes but almost normal eEJC amplitudes attributable to a compensatory increase in presynaptic vesicle release (Petersen et al., 1997; DiAntonio et al., 1999). We also analyzed a mutant with genetically elevated subsynaptic protein synthesis [poly(A)binding protein $(p a b p)$ ], which showed unaltered quantal size but strongly increased eEJCs caused by enhanced evoked vesicle release (Sigrist et al., 2000). Our results from both mutants provide evidence that a persistently enhanced evoked release of 
presynaptic vesicles is achieved primarily by recruiting additional active zones. In dglurII $A$ - $k o$ mutants, these additional active zones were distributed in a smaller number of boutons, resulting in an increased active zone density, larger evoked presynaptic $\mathrm{Ca}^{2+}$ signals, and rescued junctional transmission of single stimuli but considerably depressed transmission of repetitive signals. In contrast, in $p a b p^{P 970} /+$ mutants, the additional active zones were equally distributed in newly grown boutons resulting in a similar active zone density and evoked $\mathrm{Ca}^{2+}$ signals as in wildtype animals and an improved junctional reliability after repetitive stimulation.

\section{MATERIALS AND METHODS}

Genetics. The glutamate receptor alleles $\operatorname{dglurII} A^{g 9}$ and $\mathrm{df}(2 \mathrm{~L}) \mathrm{clh} 4$ have been described in Petersen et al. (1997), and the alleles of the poly(A)binding protein gene ( $p a b p^{P 970}, p^{P a b p^{E P 0310}}$, and $\left.\mathrm{df}(2 \mathrm{R}) \mathrm{Pcl} 7 \mathrm{~b}\right)$ have been described in Sigrist et al. (2000). Transgenic UAS-yCam2 flies were generated by inserting the yCam2-cDNA (Miyawaki et al., 1997) into the pUAST vector and P-element-mediated transformation of Drosophila (Berkeley Drosophila Genome Project). For all experiments we used the line $\mathrm{P}\left(\mathrm{w}^{+}\right.$; UAS-yCam 2$) 2-6 \mathrm{i}$ as yCam 2 donor. yCam2 expression was driven in all larval neurons using the Gal4-driver lines (Brand and Perrimon, 1993) elav-Gal4, and C155-Gal4 (generous gift of C. S. Goodman, University of California at Berkeley, Berkeley, CA). The following male genotypes have been analyzed: wt: wild type. Elav-Cam: +/Y;elavGal4/+;P2-6i/+. C155-Cam: C155Gal4/Y;+/+;P2-6i/+. Elav-Campabp: +/Y;elavGal4/pabp ${ }^{P 970} ; \mathrm{P} 2-6 \mathrm{i} /+$. C155-CamdglurIIA: $\mathrm{C} 155 / \mathrm{Y} ;$ dglurII $A^{\text {g9 }} / \mathrm{df}(2 \mathrm{~L}) \mathrm{clh} 4 ; \mathrm{P} 2-6 \mathrm{i} /+$.

Larval culture. All larvae were raised under tightly controlled culture conditions (high-density culture, $65 \%$ humidity) at $28^{\circ} \mathrm{C}$ to increase Gal4 and thus yCam2 expression, except for one population of wild-type animals that was raised at $29^{\circ} \mathrm{C}$. Mid-third instar male larvae were selected before the wandering stage, dissected in $\mathrm{Ca}^{2+}$-free HL3 solution (Stewart et al., 1994), and pinned onto a Sylgard-coated coverslip.

Electrophysiology. Two-electrode voltage-clamp recordings were performed in $1 \mathrm{~mm}$ extra cellular $\mathrm{Ca}^{2+}$ (HL3) as described (Sigrist et al., 2000, 2002). Voltage errors were $<3 \mathrm{mV}$ for eEJCs of $\geq 100 \mathrm{nA}$. Nerve stimuli were applied at a frequency of $0.2 \mathrm{~Hz}$, and 30-50 eEJCs were averaged per muscle (clamped at $-60 \mathrm{mV}$ ). All cells had comparable resting potentials between -65 and $-75 \mathrm{mV}$. The input resistance $(\sim 5$ $\mathrm{M} \Omega$ ) was unchanged in all genotypes except in control C155-Cam animals, which showed a slight but significant decrease compared with all other genotypes $(p<0.002)$. For miniature EJC (mEJC) recordings, cells were clamped to $-70 \mathrm{mV}$ for $120 \mathrm{sec}$. Signals were analyzed by use of commercial software (Jaejin Software; Synaptosoft, Decatur, GA). Evoked excitatory junction potential (eEJP) amplitudes during spiketrain stimulation experiments were obtained by fitting the falling phase of the leading eEJP to a double exponential and extrapolating it to the time point of the following peak. The extrapolated base of the leading eEJP was subtracted from the peak value of the following eEJP. Junctional depression was analyzed by dividing the amplitude of the ninth eEJP by that of the first eEJP of a given stimulus train.

$y$ Cam 2 imaging. The enhanced cyan fluorescent protein (ECFP) module of yCam2 was excited at $435 \pm 10 \mathrm{~nm}$ using a monochromatic light source (TILL-Photonics). Emitted light was separated from excitation by a dichroic mirror at $460 \mathrm{~nm}$. A second dichroic mirror at $505 \mathrm{~nm}$ (ratio) or $515 \mathrm{~nm}$ (single wavelength) and two band pass filters at $485 \pm 20$ and $540 \pm 25 \mathrm{~nm}$ were used to isolate the $485 \mathrm{~nm}$ ECFP and the $530 \mathrm{~nm}$ enhanced yellow fluorescent protein (EYFP) emissions (see Fig. 1C). Two CCD cameras (PXL, Photometrics, Tucson, AZ), the monochromator device, and the image analysis were controlled using IPLab (Scanalytics, Fairfax, VA). Action potentials were evoked time locked to image acquisition. To prevent muscle movements during imaging, dissected larvae were preincubated in HL3 containing $1 \mu \mathrm{M}$ thapsigargin for $40 \mathrm{~min}$. Imaging experiments were performed in HL3 containing $1 \mu \mathrm{M}$ thapsigargin and $1.5 \mathrm{~mm}$ glutamate to desensitize junctional glutamate receptors. Single wavelength recordings were achieved as follows: $40 \times$ objective, $2 \times 2 \mathrm{CCD}$ binning, i.e., $750 \times 750 \mathrm{~nm}$ per hyperpixel, 116 msec per frame $(128 \times 64$ hyperpixels $)$; dual-wavelength recordings were achieved as follows: $60 \times$ objective, unbinned CCDs, i.e., $250 \times 250 \mathrm{~nm}$ per pixel, $500 \mathrm{msec}$ per frame $(256 \times 128$ pixels $)$ and camera.

Stacks of raw data images were first linearly corrected for bleaching. Subsequently, the background measured in nonjunctional regions was subtracted locally. These image stacks were converted into $\Delta F / F$ movies, where $F$ is the average fluorescence of several frames taken before nerve stimulation and $\Delta F$ is the signal deviation from $F$ in every image. For quantitative analyses, $\Delta F / F$ changes of selected junctional regions were plotted individually or averaged as a function of the experimental time course. For presentation purposes, $\Delta F / F$ changes were color encoded using a linear color gradient. Image data from simultaneous dual emission imaging experiments were similarly corrected for bleaching and local background before the EYFP/ECFP ratios were calculated $(\Delta R / R$; $R$, average EYFP/ECFP ratio of several frames taken before nerve stimulation; $\Delta R$, ratio deviation from $R$ in every image). Because of massive muscle contractions during the calibration procedure, we were not able to quantify $\mathrm{Ca}^{2+}$ concentrations in this system.

For the comparison of different genotypes, junctional regions of interest (ROIs) were selected from raw fluorescence images of muscle 6/7 in abdominal segment 2 (see Fig. 2 A, blue circles). ROIs were preferentially placed into larger boutons (average ROI, $4.8 \mu \mathrm{m}^{2}$ ); however, $15 \%$ of all analyzed ROIs were between 1 and $3 \mu \mathrm{m}^{2}$. Muscle $6 / 7$ is typically innervated by axons from two different motoneurons that give rise to anatomically and physiologically distinguishable boutons of type $\mathrm{Ib}(2-5$ $\mu \mathrm{m}$ diameter) or Is (1-3 $\mu \mathrm{m}$ diameter) (Atwood et al., 1993; Kurdyak et al., 1994; Karunanithi et al., 1997; Lnenicka and Keshishian, 2000). Thus, ROIs are likely to reflect type Ib boutons, but type Is boutons were also selected. Linear regression analysis of the peak $\Delta R / R$ at $40 \mathrm{~Hz}$ stimulation showed no correlation to the size of the respective ROIs $(R<0.1)$.

Quantification of junction size. The number of synaptic boutons per NMJ (muscle 6/7, abdominal segment 2) was counted in Fasciclin IIlabeled (mAB1D4) larval preparations at the microscope. These raw bouton counts were normalized to the inner muscle surface area to compensate for small developmental differences of the analyzed individuals. All quantifications were performed double blind.

Electron microscopy. Larvae were filleted and processed for ultrastructural analysis as described (70 min fixation in ice-cold $4 \%$ paraformaldehyde/PBS) (Sigrist et al., 2002). Ultrathin sections from random positions of 4-16 neuromuscular branches (muscle 6/7, abdominal segment A2) of two animals per genotype were analyzed at 21,000-fold enlargement with a CM10 electron microscope (Philips). The mean number of dense areas (synaptic profiles) and dense bodies (profiles of T-bars) in sections per branch was scored. The perimeter and area of bouton profiles were measured. From these raw data we calculated the ratio of synaptic profiles with or without T-bars, the mean perimeter/area by which bouton profiles are represented in sections, and the relation of the number of T-bar profiles in a bouton profile to its volume (area $\times$ thickness of a section $87 \mathrm{~nm}$ ) or its perimeter.

\section{RESULTS}

\section{Targeted transgenic expression of the $\mathrm{Ca}^{2+}$ sensor yCam2 in Drosophila neurons}

To study presynaptic $\mathrm{Ca}^{2+}$ dynamics in single boutons of NMJs, we developed for Drosophila an alternative approach to the so far successfully applied $\mathrm{Ca}^{2+}$ imaging in this system that was based on synthetic $\mathrm{Ca}^{2+}$ sensors (Karunanithi et al., 1997; Umbach et al., 1998a,b; Dawson-Scully et al., 2000; Bronk et al., 2001). This new approach relies on the targeted transgenic expression of the $\mathrm{Ca}^{2+}$ sensor protein yellow cameleon-2 (Miyawaki et al., 1997; Kerr et al., 2000), which offers access to cells and cellular compartments that are difficult or impossible to approach using injection or general loading of synthetic dyes (see Discussion). To bypass such limiting factors we created transgenic flies with a P-element transformation vector containing the yellow Cameleon-2 (yCam2) cDNA (Miyawaki et al., 1997) downstream of an array of UAS sites. This allowed the targeted expression of yCam2 in all cells expressing the yeast transcriptional enhancer Gal4 (Brand and Perrimon, 1993). In this study, we used two Gal4-driver lines (elav-Gal4 and C155-Gal4) that both led to a reliable and strong expression of yCam2 in all larval neurons. yCam2 highlighted the entire larval brain, the motor nerves (Fig. 1 $A$ ), and junctional nerve terminals at body wall muscles in the larval periphery (Fig. $1 B$ ). It is important to note that this transgenic expression of yCam2 was specific for neuronal structures, such as 


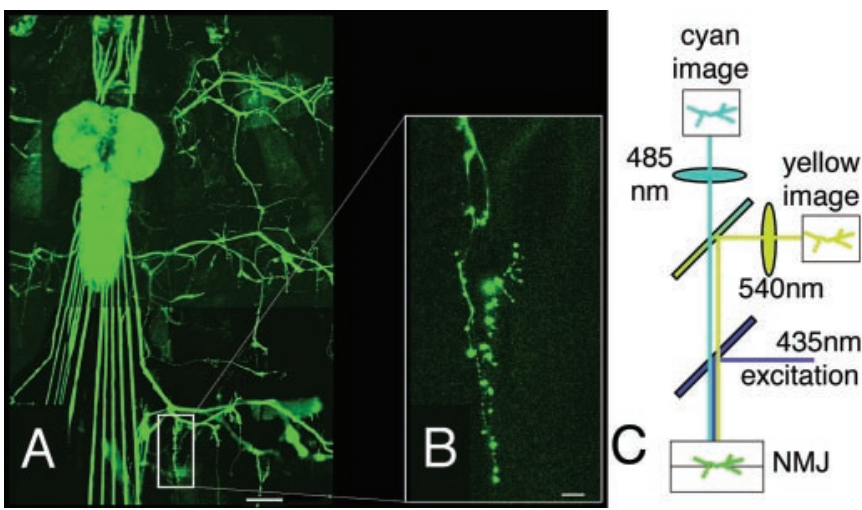

Figure 1. Transgenic expression of the $\mathrm{Ca}^{2+}$ sensor molecule yCam2 in Drosophila neurons. $A$, yCam2 fluorescence in elav-Cam transgenes (see Materials and Methods) highlights all neurons in the larval brain, including motoneurons, their axonal projections to the hemisegments of the periphery, and the presynaptic terminals of NMJs at the larval body wall musculature $(B)$. Note the lack of fluorescence in non-neuronal tissues. Scale bars: $A, 100 \mu \mathrm{m} ; B, 10 \mu \mathrm{m}$. $C$, Simultaneous dual emission recording of yCam2 was performed by exciting yCam2 in presynaptic terminals of larval NMJs at $435 \mathrm{~nm}$ and isolating the emission fluorescence of ECFP and EYFP using a beam splitter and appropriate filters (see Materials and Methods). ECFP and EYFP fluorescence signals were recorded with two synchronously operated CCD cameras.

boutons at NMJs, and that fluorescence signals were absent from muscles or other non-neuronal tissues (Figs. 2, 3).

After $\mathrm{Ca}^{2+}$ binding, yCam2 undergoes a conformational change, which brings its two chromophores ECFP and EYFP into close proximity (Miyawaki et al., 1997). This greatly increases the probability of fluorescence resonance energy transfer (FRET) from excited ECFP to EYFP, resulting in an increased EYFP $(530 \mathrm{~nm})$ emission and a corresponding decrease of the ECFP $(485 \mathrm{~nm})$ emission. The FRET-based radiometric nature of this $\mathrm{Ca}^{2+}$ indicator makes it suitable for imaging in whole animal preparations, because it reduces the susceptibility to motion artifacts and greatly increases the accuracy of the measured fluorescence changes (Fan et al., 1999; Kerr et al., 2000). We therefore recorded the emission intensities of ECFP and EYFP (Fig. $1 C$ ), either in consecutive stimulation trials to analyze the basic yCam2 properties (Fig. 2) or simultaneously and thus more accurately to analyze synaptic parameters in various genotypes (Figs. 3, 6).

\section{yCam2 is a reliable $\mathrm{Ca}^{2+}$ sensor in Drosophila neurons}

A considerable problem of presynaptic $\mathrm{Ca}^{2+}$ imaging at NMJs was that the postsynaptic muscle cell starts to contract, in particular during high-frequency (HF) stimulation. To prevent muscle contraction during nerve stimulation, we treated the larval preparations with bath-applied glutamate to desensitize postsynaptic glutamate receptors. To further suppress excitation-contraction coupling, we used thapsigargin to deplete the intracellular $\mathrm{Ca}^{2+}$ stores (Thastrup et al., 1990). These treatments effectively eliminated muscle contraction even during bursts of high-frequency stimulation. In addition, thapsigargin blocks the function of presynaptic $\mathrm{Ca}^{2+}$ stores at Drosophila NMJs (Kuromi and Kidokoro, 2002) and thus reveals the dynamics of evoked presynaptic $\mathrm{Ca}^{2+}$ influx without the functional contribution of intracellular stores. Using this preparation and the described setups (see Materials and Methods), we observed opposing dynamics of ECFP and EYFP emission intensities in presynaptic terminals of larval NMJs during periods of nerve stimulation (Fig. $2 B, C$ ), demon-
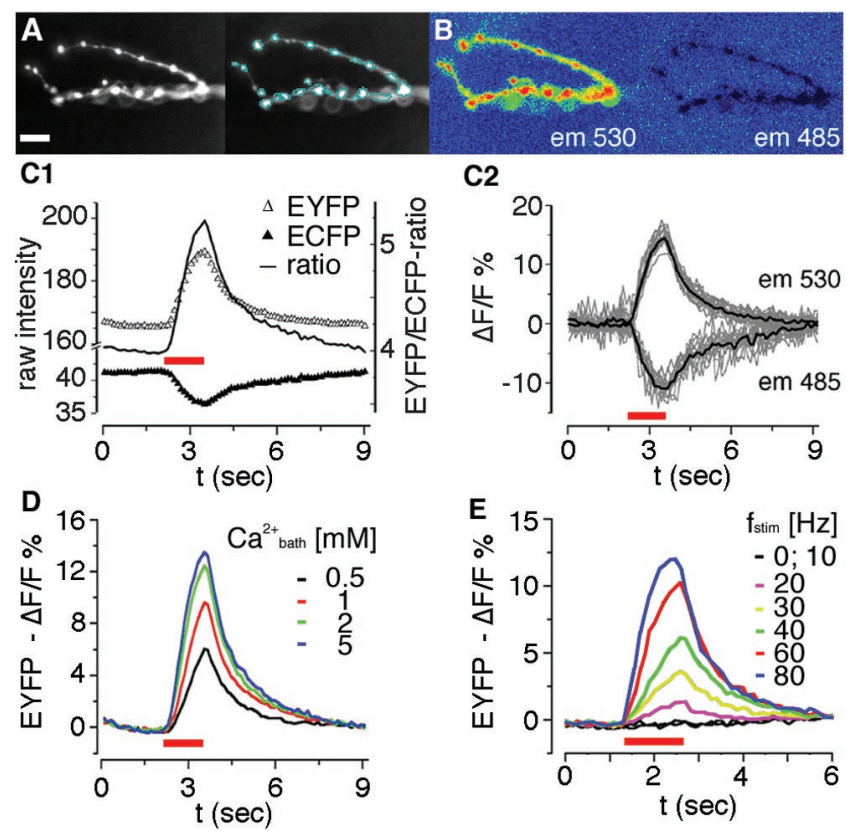

Figure 2. Presynaptic $\mathrm{Ca}^{2+}$ imaging at Drosophila NMJs using transgenically expressed yCam2. $A$, yCam2 fluorescence in elav-Cam transgenes highlights all presynaptic terminals of larval NMJs. Boutons within the focal plane of the image (blue-circled areas) were selected for quantitative analysis. Scale bar, $15 \mu \mathrm{m}$. B, False color images encode positive and negative fluorescence changes of EYFP (530 nm) and ECFP (485 nm) during nerve stimulation, which are indicative of FRET between excited ECFP and EYFP. $C-E$, Quantitative analysis of yCam2 fluorescence changes during spike-train stimulation (red bars). C1, Consecutive recordings of the $\operatorname{EYFP}(\triangle)$ and $\operatorname{ECFP}(\boldsymbol{\Delta})$ emission intensities show opposing signal changes during stimulation $\left(40 \mathrm{~Hz}, 1.3 \mathrm{sec} ; 5 \mathrm{mM} \mathrm{Ca}^{2+}\right)$ resulting in an EYFP/ECFP ratio change of $28 \%$ (line). $C 2$, All individual boutons marked in $A$ show almost identical evoked fluorescence changes (thin lines), which on average (thick lines) reach $\Delta F / F$ peak values of $+14.4 \pm$ $0.5 \%$ (EYFP) and $-11.0 \pm 0.4 \%$ (ECFP). EYFP emission changes depend on extracellular $\mathrm{Ca}^{2+}$ concentration (stimulus: $40 \mathrm{~Hz}, 1.3 \mathrm{sec} ; 119$ boutons; 6 animals) $(D)$ and on the frequency of presynaptic spike trains (stimulus: $1.3 \mathrm{sec} ; 1 \mathrm{mM} \mathrm{Ca}^{2+} ; 58$ boutons; 4 animals) $(E)$.

strating that the shown EYFP emission alterations in Figure 2, $D$ and $E$, are caused by FRET. Spike train-evoked fluorescence changes displayed homogeneous time courses and peak amplitudes for all analyzed junctional areas (Fig. $2 A$, blue circles, $C 2$, thin lines) independent of their size and location within the NMJ (see below). Evoked yCam2 fluorescence changes faithfully reflected alterations in extra cellular $\mathrm{Ca}^{2+}$ concentrations (Fig. 2D) and presynaptic frequency of action potentials (Fig. 2E). Simultaneous measurements of both emission wavelengths (Fig. 3A,B) greatly improved the signal-to-noise ratio and allowed us to reliably quantify yCam 2 fluorescence changes on a single bouton level (Fig. 3B,C). We therefore applied simultaneous dualemission imaging to analyze and compare presynaptic $\mathrm{Ca}^{2+}$ dynamics among several animals and genotypes (see Fig. 6). These experiments were performed at stimulation frequencies $(40 \mathrm{~Hz})$ and an extra cellular $\mathrm{Ca}^{2+}$ concentration $(1 \mathrm{~mm})$ that evoked fluorescence changes well within the observed dynamic range of yCam2 (Fig. 2). Taken together, these results demonstrate that the Gal4-mediated expression of yCam2 in Drosophila neurons represents a noninvasive and sensitive tool to monitor spike train-evoked alterations of presynaptic $\mathrm{Ca}^{2+}$ at junctional boutons.

It is interesting to note that the monitored evoked fluorescence 

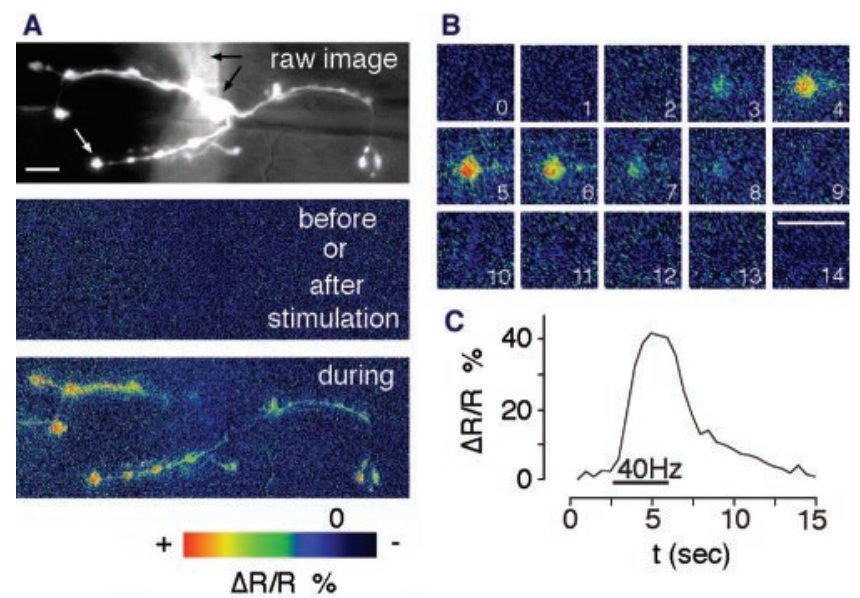

Figure 3. Simultaneous dual emission imaging allows a reliable analysis of yCam2 fluorescence changes on a single bouton level. A, Top panel shows a raw image of a yCam2-expressing NMJ (C155-CamdglurIIA). Black arrows mark an underlying nerve. The false color images encode EYFP/ECFP ratio changes ( $\Delta R / R$; see Materials and Methods) before or after and during nerve stimulation $\left(40 \mathrm{~Hz} ; 3.5 \mathrm{sec} ; 1 \mathrm{mM} \mathrm{Ca}^{2+}\right)$. Note that $\Delta R / R$ changes were detected only in presynaptic boutons and not in yCam2-expressing nerves and axons or areas outside of NMJs. $B$, The time course of $\Delta R / R$ changes is represented for a single bouton $(A$, white arrow) in a series of false color images (time in seconds is indexed) or plotted in $C$. We detected a rapid onset of $\Delta R / R$ changes that reached up to $40 \%$ on a single bouton level. Scale bars: $A, 6 \mu \mathrm{m} ; B, 10 \mu \mathrm{m}$.

alterations of yCam2 showed no correlation to the size of the analyzed ROIs $(R<0.1$; see Materials and Methods), indicating that the bouton size has no detectable effect on the evoked $\mathrm{Ca}^{2+}$ signal. This finding also suggested that the arborizations of the two innervating motoneurons of muscle $6 / 7$, which can be anatomically and physiologically differentiated in large type Ib boutons (2-5 $\mu \mathrm{m}$ diameter) and smaller type Is boutons $(1-3 \mu \mathrm{m})$ (Atwood et al., 1993; Kurdyak et al., 1994), show similar evoked $\mathrm{Ca}^{2+}$ dynamics. A similar observation has been made previously in this system using the synthetic $\mathrm{Ca}^{2+}$-sensitive dye fluo-3 AM in conjunction with confocal microscopy (Karunanithi et al., 1997); the authors suggested that factors other than differences in presynaptic $\mathrm{Ca}^{2+}$ entry may govern the physiological differences between both bouton types.

\section{Expression of yCam2 does not affect the physiological and morphological development of wild-type and mutant NMJs}

The $\mathrm{Ca}^{2+}$-binding module of $\mathrm{yCam} 2$ is derived from the calciumbinding protein calmodulin (Miyawaki et al., 1997) and binds $\mathrm{Ca}^{2+}$ ions effectively over a broad range of physiological concentrations. This $\mathrm{Ca}^{2+}$-buffering capacity of yCam2 raised the concern that strong transgenic overexpression of yCam 2 could act as an additional intracellular $\mathrm{Ca}^{2+}$ buffer that may affect the physiology and development of yCam2-expressing cells and animals. We therefore examined the physiological and morphological development of NMJs with and without neuronal yCam2 expression. First we analyzed the basal junctional signal transmission by measuring the postsynaptic input resistance (Fig. $4 C$ ) and mEJCs and eEJCs (Fig. 4A,B). From these data we calculated the junctional quantal content (mean eEJC divided by mean mEJC) (Fig. $4 C$ ) as an estimate of the number of vesicles that are released in response to a presynaptic action potential. In addition we quantified the number of presynaptic boutons per NMJ (Fig. 5A,B) and examined the relationship between the number of boutons
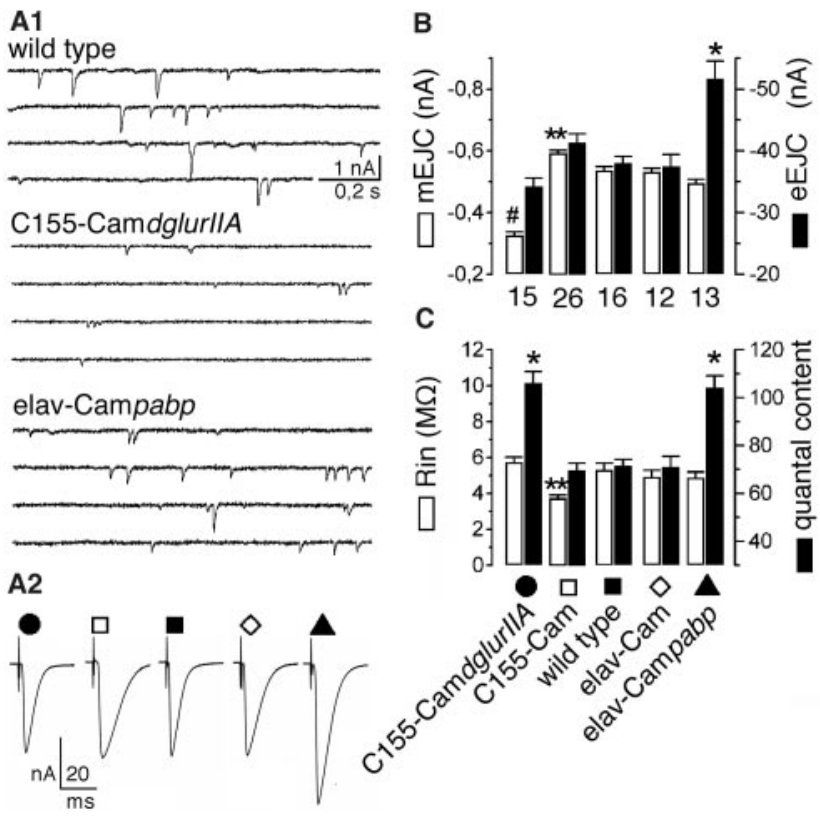

Figure 4. yCam2 expression in larval neurons does not affect the junctional physiology of wild-type and mutant animals. Representative traces of mEJCs $(A 1)$ and eEJCs $(A 2)$ of the symbolized genotypes $(C) . B, C$, yCam2 expression did not alter the amplitudes of mEJCs or eEJCs in wild-type controls [elav-Cam $(\diamond)$; wild type $(\square)$ ]. The derived junctional quantal contents (mean eEJC divided by mean mEJC) therefore remained unchanged $(p>0.5)$. Likewise, yCam2-expressing mutant animals showed similar phenotypes as reported previously for the mutants themselves. C155-CamdglurIIA NMJs (O) have significantly reduced mEJC amplitudes ( $\left.{ }^{*} p \ll 0.0001\right)$, unaltered eEJC amplitudes, and an increased junctional quantal content compared with C155-Cam controls $\left({ }^{*} p<0.001\right)$. elav-Campabp NMJs $(\boldsymbol{\Delta})$ show unaltered mEJCs, significantly larger eEJCs, and thus an increased junctional quantal content compared with elav-Cam controls $\left({ }^{*} p \ll 0.001\right)$. Note that in both mutants the junctional quantal content and thus the rate of evoked vesicle release are similarly increased relative to wild type. The muscle input resistance $\left(R_{\mathrm{in}}\right)$ in C155-CamdglurII $A$ and elav-Campabp is similar to that of wild-type animals $(p=0.35)$. The decreased $R_{\text {in }}$ of C155-Cam $(\square)$ muscles compared with wild type and the associated slight increase in $\operatorname{mEJC}(* * p<0.002)$ and eEJC amplitudes is likely attributable to the somewhat larger muscle sizes of C155 animals (our unpublished observations). The number of analyzed cells is shown in $B$.

(NMJ size) and junctional quantal content (Fig. 5C). All of the above parameters showed no detectable difference between wildtype animals expressing yCam2 (elav-Cam) and those that did not (Fig. 4B,C, wild type). The decreased input resistance of muscles in C155-Cam animals and the associated slight increase in $\mathrm{mEJC}$ and eEJC amplitudes were likely caused by the somewhat larger muscle size of animals harboring the C155chromosome (our unpublished observations); however, these alterations did not affect the quantal content of these NMJs (Fig. $4 C)$. Thus, in accordance with a similar analysis performed in Caenorhabditis elegans (Kerr et al., 2000), strong yCam2 expression does not interfere with the cellular physiology or development of Drosophila NMJs.

We extended this analysis to two additional genotypes, both of which showed a similarly increased evoked release of presynaptic vesicles: animals with elevated subsynaptic translation $\left(\right.$ pabp $\left.^{P 970} /+\right)$ (Sigrist et al., 2000) and animals with a postsynaptic defect attributable to the loss of the gene encoding the glutamate receptor subunit DGluR-IIA (dglurII $A^{A D 9}$ / df(2L)clh4) (Petersen et al., 1997). We found that the transgenic 

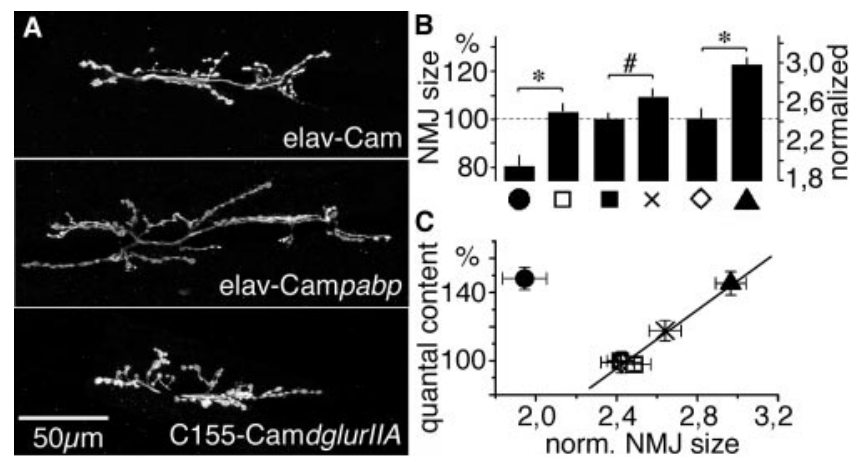

Figure 5. Typical and atypical relationship between the strength and size of NMJs. $A, B$, A morphological analysis of Fasciclin II-labeled NMJs (see Materials and Methods) revealed that elav-Campabp NMJs $(\boldsymbol{\Delta} ; n=27)$ develop more boutons and C155-CamdglurIIA animals $(\mathbf{0} ; n=17)$ develop fewer boutons than control larvae [elav-Cam $(\diamond ; n=43)$; C155-Cam ( $\square$; $n=21)$, wild type $\left.(\square ; n=33) ;{ }^{*} p \ll 0.001\right]$, all of which have been reared under normalized culture conditions. Raising of wildtype larvae at $29^{\circ} \mathrm{C}(\times ; n=39)$ resulted in a small but significant increase of NMJ size compared with animals reared at $28^{\circ} \mathrm{C}\left(\mathbf{\square} ;{ }^{\#} p<0.05\right)$. $C$, Consistent with previous findings without $y \mathrm{Cam} 2$ expression, the strength of junctional signal transmission (junctional quantal content) correlated significantly with the size of NMJs $(r=0.98)$. This tight structurefunction relationship was disrupted in C155-CamdglurIIA larvae (-), which showed elevated vesicle release from fewer presynaptic boutons. All data are plotted as means \pm SEM.

expression of yCam2 did not again affect the junctional phenotypes known from both mutants; $p a b p^{P 970} /+$ larvae that expressed yCam2 in all neurons (elav-Campabp) showed a typical strengthening of junctional signal transmission (Sigrist et al., 2000) as measured by larger eEJC amplitudes and unaltered mEJC size (Fig. $4 B$ ), resulting in a significantly increased junctional quantal content (Fig. 4C). elav-Campabp NMJs had also significantly more boutons compared with yCam2-expressing controls (elav-Cam) (Fig. 5B), whereas the muscle size and the muscle $R_{\text {in }}$ remained unaltered (Fig. $4 C$ ). Likewise, as reported previously for the DGluR-IIA-knock-out mutant alone (Petersen et al., 1997; DiAntonio et al., 1999), C155-CamdglurII $A$ animals showed a reduction of the average quantal size (mEJCs) and normal eEJC amplitudes (Fig. 4B), resulting in a similarly increased junctional quantal content $(150 \%)$ as seen at elavCampabp NMJs (Fig. 4C). Under the larval rearing conditions used in this study (see Materials and Methods), C155CamdglurII $A$ animals and DGluR-IIA-knock-out mutants developed significantly smaller NMJs on muscles with unaltered $R_{\text {in }}$ than all other genotypes examined in this study (Fig. 5A,B). Thus, it appears that the yCam 2 expression in all neurons does not interfere with the physiological or morphological development of even mutant NMJs and therefore allows a detailed analysis of presynaptic $\mathrm{Ca}^{2+}$ dynamics in such mutant animals.

It is important to note that the junctional size and strength of elav-Campabp larvae and wild-type animals showed a characteristic proportional relationship (Fig. $5 C$ ), which has been described in several recent studies with independent genotypes (Cheung et al., 1999; Sigrist et al., 2000, 2002; Sanyal et al., 2002). NMJs with genetically strengthened signal transmission and unaltered muscle input resistance $\left(R_{\mathrm{in}}\right)$ are proportionally larger than control NMJs (Sigrist et al., 2000). C155-CamdglurII $A$ animals, which apparently compensate for their postsynaptic defects by releasing more vesicles, represent an exception to this structure-function relationship (Fig. $5 C$, filled circle): during stimulation, these mu-
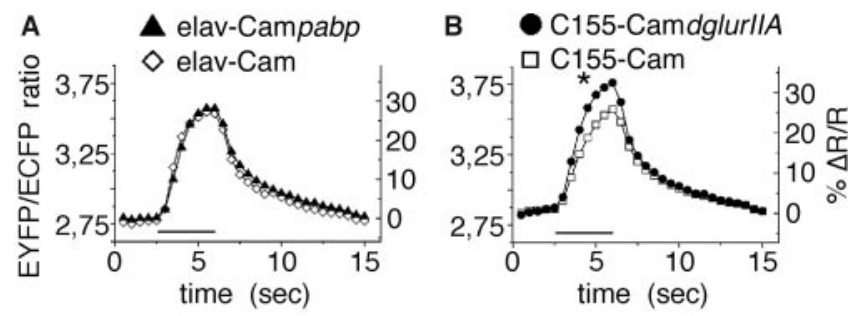

Figure 6. Comparison of spike train-evoked presynaptic $\mathrm{Ca}^{2+}$ signals from NMJs of various genotypes. $A$, Simultaneous EYFP-ECFP imaging revealed that stimulation-evoked presynaptic $\mathrm{Ca}^{2+}$ dynamics (stimulus: $40 \mathrm{~Hz}, 3.5 \mathrm{sec}, 1 \mathrm{~mm} \mathrm{Ca}^{2+}$ ) are indistinguishable at elav-Campabp NMJs (195 boutons, 7 animals) and elav-Cam NMJs (130 boutons, 7 animals). Note that these genotypes differ in their junctional transmission strength, but they conform to the described correlation of junctional size and strength. $B$, The same stimulation protocol elicited a significantly larger EYFP/ECFP ratio change at C155-CamdglurIIA NMJs (318 boutons, 14 animals) compared with C155-Cam controls (283 boutons, 13 animals; ${ }^{*} p \ll 0.001$ ). Data are given as means \pm SEM (error bars are hidden within the symbols).

tants release an increased number of vesicles from a significantly smaller NMJ. These obvious differences allowed us to address the question of how Drosophila NMJs may mediate persistently enhanced presynaptic vesicle release in different genotypes.

\section{Larger evoked $\mathrm{Ca}^{2+}$ signals in presynaptic boutons of C155-CamdglurIIA NMJs compared with elav- Campabp and wild-type animals}

Because evoked vesicle release relies on stimulus-induced changes in presynaptic $\mathrm{Ca}^{2+}$ influx (Mallart, 1993; Tank et al., 1995; Feller et al., 1996; Sinha et al., 1997; Catterall, 1998; Zucker, 1989, 1999), we hypothesized that the enhanced vesicle release in the above two mutants could be caused by an increased $\mathrm{Ca}^{2+}$ influx at preexisting active zones (Mallart, 1993) or by an increased sensitivity of the presynaptic release machinery to $\mathrm{Ca}^{2+}$ (Dawson-Scully et al., 2000). Alternatively, a larger number of released vesicles could also arise from a larger total number of active release sites per NMJ. Given that the mutants showed a similarly enhanced evoked vesicle release compared with wildtype NMJs but large differences in junctional bouton numbers, we attempted to differentiate between these possibilities by examining the spike train-evoked presynaptic $\mathrm{Ca}^{2+}$ dynamics in these animals (Fig. 6).

Simultaneous EYFP/ECFP emission ratios $(\Delta R / R)$ of randomly chosen boutons showed an identical baseline before stimulation in all analyzed genotypes (Fig. 6), suggesting that there were no substantial differences in resting $\mathrm{Ca}^{2+}$ levels of presynaptic terminals among all examined animals. During stimulation (Fig. 6, black lines), the mean ratio changes observed at boutons of elav-Cam and elav-Campabp animals were indistinguishable (Fig. 6A), indicating that stimulus-induced presynaptic $\mathrm{Ca}^{2+}$ changes were similar in both genotypes. This observation demonstrates that the enhanced junctional signal transmission measured at elav-Campabp NMJs does not rely on a persistent increase in presynaptic $\mathrm{Ca}^{2+}$ dynamics. Because all analyzed junctional boutons showed similar $\mathrm{Ca}^{2+}$ signals during stimulation, it rather appeared that the larger number of released presynaptic vesicles seen in this mutant (Fig. $4 C$ ) could be attributable to the larger number of similarly contributing boutons $(125 \%$ NMJ size) (Fig. 5C, triangle). This interpretation is consistent with the above-mentioned relationship of evoked junctional vesicle release and NMJ size, and it implies that long-term strength- 
ening of signal transmission at larval NMJs may be mediated primarily by the addition of active zones that are distributed in newly grown junctional boutons.

In contrast, yCam2 imaging in boutons of C155-CamdglurIIA animals revealed that $\Delta R / R$ values were increased significantly during nerve stimulation compared with controls $(127 \%)$ or other genotypes (Fig. 6). Given that the imaged presynaptic terminals of mutant and control animals showed no detectable differences in resting $\mathrm{Ca}^{2+}$ levels before and after stimulation, these data suggest that spike train-evoked $\mathrm{Ca}^{2+}$ influx into junctional boutons is strongly enhanced in C155-CamdglurII $A$ animals. This result indicates that either the evoked $\mathrm{Ca}^{2+}$ influx at given release sites is enhanced or that the number of responsive release sites within each bouton is increased. In addition it demonstrates for the first time that the compensation for the defective postsynaptic glutamate receptor function, which leads to an increased evoked vesicle release (Petersen et al., 1997; DiAntonio et al., 1999), is likely mediated by a retrograde signaling mechanism that results in an elevation of evoked $\mathrm{Ca}^{2+}$ influx into junctional boutons.

As mentioned above, the DGluR-IIA-knock-out mutant represents an exception to the structure-function relationship seen in several other genotypes (Cheung et al., 1999; Sigrist et al., 2000, 2002; Sanyal et al., 2002) because this mutant releases more vesicles ( $150 \%$ junctional quantal content) from a reduced number of boutons ( $80 \%$ NMJ size) (Fig. $5 C$, circle). The increased presynaptic $\mathrm{Ca}^{2+}$ influx per bouton is therefore compatible with the above idea that elevated evoked vesicle release may be mediated by an increased number of active zones, which in this genotype would be packed into a smaller number of boutons. Alternatively, because presynaptic vesicle release increases roughly with the third power of presynaptic $\mathrm{Ca}^{2+}$ (Tank et al., 1995; Feller et al., 1996), the elevated junctional quantal content of this mutant also may be linked to an upregulation of presynaptic $\mathrm{Ca}^{2+}$ dynamics at preexisting active zones. To differentiate between these possibilities, we performed an ultrastructural examination of NMJs of the genotypes used here.

\section{Increased number of active zones at NMJs with enhanced vesicle release}

A large body of evidence has suggested that NMJs of Drosophila larvae harbor a heterogeneous set of synapses that differ in their probability of presynaptic vesicle release (Atwood et al., 1993; Wojtowicz et al., 1994; Atwood and Wojtowicz, 1999). Synapses with a high probability of vesicle release appear to harbor presynaptic T-shaped dense bodies ("T-bars") and were therefore termed "active zones" (Cooper et al., 1995, 1996), whereas T-barfree synapses lacked the characteristic clusters of docked synaptic vesicles and are thought to represent sites of low vesicle release probability (Atwood et al., 1993; Wojtowicz et al., 1994; Atwood and Wojtowicz, 1999). To assess whether the increased evoked vesicle release seen in the above mutants is associated with such ultrastructural alterations of the synaptic morphology, we examined ultrathin sections of boutons from wild-type animals, DGluR-IIA-ko mutants (dglurII $\left.A^{A D 9} / d f(2 L) c l h 4\right)$, and the pabpmutant $p a b p^{E P 0310} / d f(2 R) p c l 7 b$, which showed for all so far examined junctional aspects stronger phenotypes than the here used allele $\mathrm{pabp}^{P 970} /+$ (Sigrist et al., 2000). From a random set of ultrathin sections of 4-16 type Ib bouton branches per genotype (see Materials and Methods), we scored the number of synaptic profiles (Fig. $7 A$, electron-dense area between arrowheads) per section, the presence or absence of presynaptic T-bar profiles (Fig. $7 A$, arrows), and the presynaptic terminal area per section
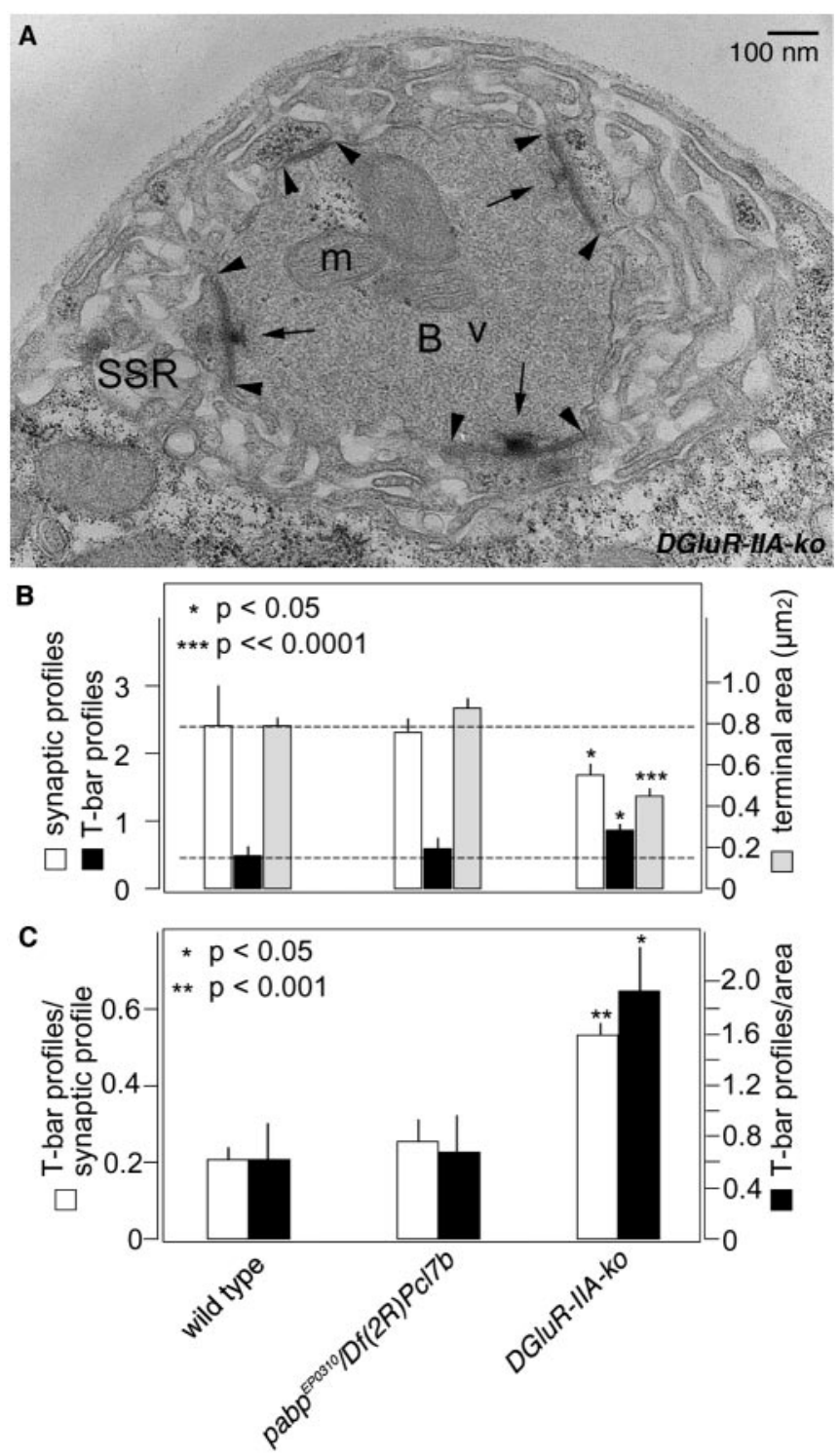

Figure 7. Comparison of the bouton ultrastructure of the indicated genotypes. $A$, Representative electron micrograph of an ultrathin section through a type Ib bouton (muscle 6 , segment A2) of a dglurIIA-ko animal (dglurII $A^{A D 9} / d f(2 L)$ clh 4 ). Note that three of the four cut synaptic profiles (electron-dense areas between arrowheads) harbor a presynaptic T-bar profile (arrows). $v$, Presynaptic vesicles; $m$, mitochondrion; SSR, subsynaptic reticulum. $B, C$, Type Ib boutons of wild-type larvae and animals with genetically increased subsynaptic protein synthesis ( $p_{a b p}{ }^{E P 0310}$ / $d f(2 R) P c l 7)$ have similar dimensions, numbers of synaptic profiles, and presynaptic T-bar profiles per scored ultrathin section $(B)$ and therefore show a similar number and density of cut active zone profiles $(C$, white and black bars, respectively; see Table 1). In contrast, dglurIIA-ko mutants showed a significant reduction in the measured presynaptic terminal area and a corresponding decrease in the number of cut synaptic profiles compared with wild-type and pab mutants. Strikingly, the number of cut presynaptic T-bar profiles was significantly larger in DGluR-IIA-ko mutants than in wild-type animals $(B)$, resulting in a strong increase in the relative number and density of active zones per sampled section $(C$, white and black bars, respectively; see Materials and Methods). Data (Table 1) are represented as branch means \pm SEM.

(area of the vesicle-filled lumen of type Ib boutons) (Fig. $7 B$, Table 1).

Our analysis revealed that the number and density of presynaptic T-bar profiles were significantly increased in sections of 
Table 1. Summary of ultrastructural analysis

\begin{tabular}{|c|c|c|c|}
\hline Genotype & $+/+$ & $\operatorname{pabp}^{E P 0310} / D f(2 R) P c l 7 b$ & $D G l u R-I I A-k o$ \\
\hline Number of branches analyzed & 4 & 5 & 16 \\
\hline Number of scored ultrathin sections $(\sim 87 \mathrm{~nm})$ & 244 & 243 & 520 \\
\hline Covered length of NMJ $(\mu \mathrm{m})$ & 21.2 & 21.1 & 45.2 \\
\hline Number of scored synaptic profiles & 628 & 561 & 872 \\
\hline Number of scored T-bar profiles & 126 & 185 & 468 \\
\hline Mean terminal area $\left(\mu \mathrm{m}^{2}\right) /$ section & $0.8 \pm 0.3$ & $0.9 \pm 0.3$ & $0.5 \pm 0.1$ \\
\hline Number of synaptic profiles/section & $2.6 \pm 0.9$ & $2.3 \pm 0.7$ & $1.7 \pm 0.3$ \\
\hline Number of T-bar profiles/section & $0.5 \pm 0.4$ & $0.8 \pm 0.5$ & $0.9 \pm 0.3$ \\
\hline Number of synaptic profiles/terminal area & $3.1 \pm 0.6$ & $2.6 \pm 0.2$ & $3.7 \pm 0.3$ \\
\hline Number of T-bar profiles/terminal area & $0.6 \pm 0.1$ & $0.7 \pm 0.1$ & $1.9 \pm 0.2$ \\
\hline Number of T-bar profiles/synaptic profile & $0.21 \pm 0.02$ & $0.25 \pm 0.05$ & $0.53 \pm 0.03$ \\
\hline Relative density of synapses $(\%)$ & $100 \pm 19$ & $84 \pm 6$ & $119 \pm 10$ \\
\hline Relative density of active zones (\%) & $100 \pm 21$ & $108 \pm 23$ & $310 \pm 24$ \\
\hline
\end{tabular}

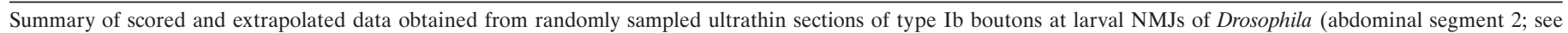

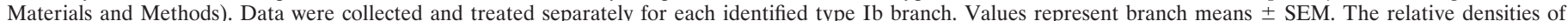

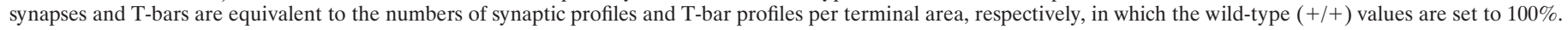

DGluR-IIA-ko mutants compared with wild-type or pabp-mutant animals, both of which showed similar values in both aspects (Fig. $7 C$, Table 1). The DGluR-IIA-ko phenotypes were attributable to a significant increase in the number of sectioned T-bar profiles, a simultaneous reduction in the average presynaptic terminal size, and a concomitant reduction in the number of synaptic profiles (Fig. $7 B$, bars on right). These data demonstrate that the number of presynaptic T-bars is subject to retrograde regulation and suggest that the persistently enhanced evoked vesicle release seen in DGluR-IIA-ko animals is caused by an increased number of active zones with high vesicle release probability. In addition, the increased density of active zones was associated with a significantly enhanced evoked $\mathrm{Ca}^{2+}$ signal in these boutons, suggesting that T-bar harboring and vesicle-loaded synapses are the sites of spike train-evoked $\mathrm{Ca}^{2+}$ influx (Umbach et al., 1998a). We therefore conclude that the increased evoked vesicle release in DGluR$I I A$ - $k o$ mutants relies on an increase in the number of active zones, which because of a junctional growth restriction are distributed at a higher density per bouton and therefore result in enhanced evoked $\mathrm{Ca}^{2+}$ signals. This interpretation is consistent with our ultrastructural, morphological, and physiological results obtained from pabp mutants. These mutants also showed an enhanced evoked vesicle release (Fig. 4) from NMJs with an increased total number of junctional boutons (Fig. 5). However, individual boutons harbored a wild-type density of active zones (Fig. 7) and thus resulted in evoked $\mathrm{Ca}^{2+}$ signals per bouton that were typical for wild-type animals (Fig. 6).

From these data we conclude that the persistently enhanced presynaptic vesicle release seen in the here-described mutants does not rely on a long-lasting increase in presynaptic $\mathrm{Ca}^{2+}$ dynamics at individual sites of vesicle release. Instead, it appears that the most important parameter is the recruitment of additional active zones. These either are packed into preexisting boutons (DGluR-IIA-ko mutants) or distributed in newly grown boutons, presumably to maintain a preferred density of active zones (wild-type animals and $p a b p$ mutants). The latter interpretation is consistent with results from a previous study performed in Drosophila and Sarcophaga (Meinertzhagen et al., 1998) that showed that active zones maintain a typical distance from each other, presumably to ensure unrestricted access to perisynaptic space.

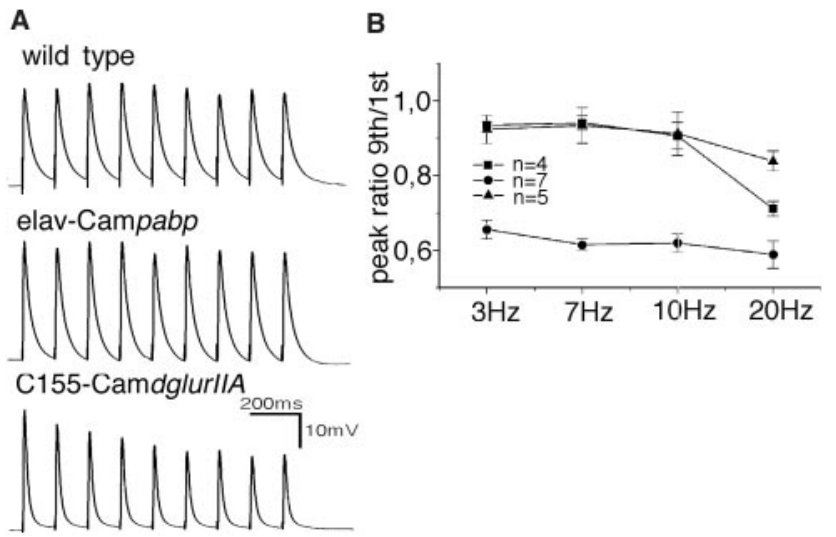

Figure 8. Comparison of junctional signal transmission of the indicated genotypes during high-frequency stimulation. $A$, Representative average traces of eEJP recordings during repetitive stimulation at $7 \mathrm{~Hz}$ (9 stimuli). $B$, NMJs of elav-Campabp animals ( $(\boldsymbol{\Delta})$ faithfully transmitted presynaptic stimuli at frequencies of up to $20 \mathrm{~Hz}$. At this frequency, wild-type NMJs (ם) showed considerable depression of postsynaptic potentials. C155CamdglurIIA animals $(\bullet)$ showed strong depression of postsynaptic potentials at all examined stimulation frequencies. Data are given as means \pm SEM.

\section{Faithful high-frequency signal transmission at larger NMJs}

The results described so far suggest that active zones and boutons represent functional compartments of NMJs that can be differentially regulated to persistently strengthen evoked vesicle release. To further assess the functional importance of junctional growth, synapse spacing, and presynaptic $\mathrm{Ca}^{2+}$, we analyzed eEJP amplitudes during repetitive synaptic signal transmission, a stimulus protocol that better resembled the neuronal activity pattern used for larval locomotion (Cattaert and Birman, 2001).

eEJP recordings during $\mathrm{HF}$ spike trains revealed that NMJs of wild-type and elav-Campabp animals performed faithful signal transmission with almost constant eEJP amplitudes at stimulation frequencies of up to $10 \mathrm{~Hz}$ (Fig. 8B). The larger NMJs of elav-Campabp animals, which also harbored a larger total number of active zones, continued to transmit without significant additional depression at higher stimulation frequencies $(20 \mathrm{~Hz})$, 
whereas wild-type NMJs showed a considerable depression of signal transmission. This form of short-term depression during HF stimulation has been analyzed extensively in this preparation (Adelsberger et al., 1997; Kuromi and Kidokoro, 1999) and in other preparations (DelCastillo and Katz, 1954; Trussell et al., 1993), and two factors have been proposed to be primarily responsible for this phenomenon: depletion of the readily releasable vesicle pool during HF stimulation (Kuromi and Kidokoro, 1999; Harata et al., 2001) and desensitization of postsynaptic neurotransmitter receptors (Trussell et al., 1993; Adelsberger et al., 1997; Heckmann and Dudel, 1997). Our above findings suggest that one or both of these factors became limiting during $20 \mathrm{~Hz}$ spike trains of wild-type NMJs, whereas NMJs of elav-Campabp animals continued to show almost constant eEJP amplitudes. It therefore appears that the addition of active zones and their distribution in additionally grown boutons ensures faithful signal transmission at high neuronal activity rates.

Interestingly, although DGluR-IIA-ko mutants release enough presynaptic vesicles on single action potentials to compensate almost completely for the postsynaptic defect of the DGluRIIA-ko mutant (Fig. 4) (Petersen et al., 1997), this compensation failed to support efficient signal transmission of higher frequency stimuli. Instead, in all tested HF stimulation patterns, these animals showed a strong depression of postsynaptic responses that tended to saturate at $\sim 60 \%$ of the initial eEJP amplitude (Fig. 8B, filled circles). A possible explanation for this enhanced depression of C155-CamdglurIIA NMJs lies in the fact that postsynaptic receptors show a strongly reduced time constant of desensitization in the absence of the DGluR-IIA subunit (DiAntonio et al., 1999). This could lead to a reduced availability of responsive receptors. In addition, the high density of active zones in this mutant and the strongly increased evoked $\mathrm{Ca}^{2+}$ influx into presynaptic boutons together could result in presynaptic $\mathrm{Ca}^{2+}$ dependent effects (Zucker, 1989, 1999; Mallart, 1993; Dittman et al., 2000), including a fast depletion of the readily releasable vesicle pool during HF stimulation (Dobrunz and Stevens, 1999; Kuromi and Kidokoro, 1999) and an enhanced competition of active zones for synaptic resources, such as the recruitment of vesicles from the reserve pool, limited vesicle recycling mechanisms, or availability of sufficient ion-buffering capacities.

Taken together our results show that the morphological consolidation of enhanced vesicle release is required to persistently strengthen junctional signal transmission. This appears to be achieved by the recruitment of T-bar harboring synapses and their equal distribution in additionally grown boutons. This ensemble of mechanisms may allow the preservation of functional properties of individual presynaptic compartments as we described for $\mathrm{Ca}^{2+}$ dynamics and active zone spacing and the strengthening of signal transmission on the junctional level. The DGluR-IIA-ko mutant may represent a genetically forced intermediate in this consolidation process, because it shows enhanced vesicle release and the recruitment of active zones. However, in the case of impaired postsynaptic glutamate receptor function, these alterations are not linked to the outgrowth of additional boutons [see also Sigrist et al. (2002)], $\mathrm{Ca}^{2+}$ dynamics are increased, and repetitive signal transmission is affected.

\section{DISCUSSION}

\section{Transgenic $\mathrm{Ca}^{2+}$ imaging in Drosophila}

The large variety of synthetic $\mathrm{Ca}^{2+}$-sensitive molecules has made them high-valued tools for physiological experiments (Grynkiewicz et al., 1985). A considerable difficulty in their experimen- tal use is the specific delivery to individual cells of interest, which is commonly achieved by invasive techniques such as dye injection or the selective uptake of a membrane-permeable derivative of the dye. This makes targeting individual small cells within complex tissues particularly difficult. To overcome such limitations, genetically encoded biosensors have been developed in recent years (Miyawaki et al., 1997, 1999; Siegel and Isacoff, 2000; Chan et al., 2001; Griesbeck et al., 2001; Honda et al., 2001; Nagai et al., 2001; Truong et al., 2001), the expression of which can be directed precisely to target tissues, individual cells, and subcellular compartments (Miyawaki et al., 1999; Griesbeck et al., 2001). In this study we used yellow Cameleon-2 (Miyawaki et al., 1997) to establish transgenic $\mathrm{Ca}^{2+}$ imaging in Drosophila and to analyze presynaptic function at larval NMJs.

Our experiments with transgenic yCam2 imaging produced results that compared well with a recent series of conventional $\mathrm{Ca}^{2+}$ imaging studies. Both approaches faithfully detected stimulation-evoked $\mathrm{Ca}^{2+}$ dynamics in boutons of Drosophila NMJs that depended on extracellular $\mathrm{Ca}^{2+}$ concentration and stimulus frequency (Fig. 2D,E) (Karunanithi et al., 1997; Umbach et al., 1998a,b; Bronk et al., 2001; Dawson-Scully et al., 2000). The spike frequency and burst duration used to elicit plateauing deviations from resting fluorescence levels were also similar (5-40 Hz for 2-5 sec) (Figs. 2, 3, 7) (Karunanithi et al., 1997; Dawson-Scully et al., 2000; Bronk et al., 2001). In accordance with Karunanithi et al. (1997), the yCam2 fluorescence fluctuations showed no correlation to the size of the analyzed boutons (Fig. 2C2) despite the pronounced differences in synaptic transmission of the larger type Ib boutons and the smaller type Is boutons (Kurdyak et al., 1994; Lnenicka and Keshishian, 2000). One difference between these conventional $\mathrm{Ca}^{2+}$ imaging experiments and our study was that we used thapsigargin to block intracellular $\mathrm{Ca}^{2+}$ store function (Thastrup et al., 1990), a treatment that thus specifically reveals the evoked $\mathrm{Ca}^{2+}$ influx from extracellular space (Kuromi and Kidokoro, 2002). Despite this, the $\mathrm{Ca}^{2+}$ imaging data were mostly comparable, suggesting that evoked presynaptic $\mathrm{Ca}^{2+}$ signals at larval NMJs originate primarily from $\mathrm{Ca}^{2+}$ influx.

Synthetic dyes generally resulted in much higher brightness levels, faster responses, and much higher evoked fluorescence changes compared with yCam2 [synthetic dyes: $250 \% \Delta F / F(\mathrm{Ka}-$ runanithi et al., 1997; Dawson-Scully et al., 2000; Bronk et al., 2001); yCam2: $40 \% \Delta R / R$ (Fig. $3 C$ )]. Therefore synthetic indicators appear to be a superior tool for detecting small $\mathrm{Ca}^{2+}$ signals in this preparation. This apparent disadvantage of using yCam2 was overcome, however, by the high specificity of the transgenic yCam 2 expression in conjunction with simultaneous ratiometric measurements. Fluorescence alterations were never detected in the subsynaptic reticulum or the surrounding muscle (Figs. 2, 3), indicating that all fluorescence signals originated in presynaptic boutons and were not contaminated by postsynaptic $\mathrm{Ca}^{2+}$ influx or muscular $\mathrm{Ca}^{2+}$ release. This feature allowed the use of standard fluorescence microscopy and CCD cameras (Fig. 1B) and may explain the high reproducibility of the recorded yCam2 fluorescence changes (Figs. 2C2, 6).

On the basis of these data obtained from the larval Drosophila NMJ, results from C. elegans (Kerr et al., 2000), and recent experiments using aequorin in mushroom body neurons of Drosophila (Rosay et al., 2001), it appears likely that the transgenic expression of recently developed genetically encoded reporter molecules will greatly facilitate future imaging studies in cells and cellular compartments that have been inaccessible so far. 


\section{Regulation of active zone number, density, and presynaptic $\mathrm{Ca}^{2+}$ dynamics during junctional development and strengthening}

In this study we addressed the question of how NMJs of Drosophila larvae achieve the continuous enhancement of evoked vesicle release seen throughout their development and during activitydependent strengthening. Using wild-type animals and two independent mutants that genetically represent both phases of junctional strengthening, we found that $\mathrm{Ca}^{2+}$-dependent presynaptic mechanisms, which are known to result in fast and reversible modifications of presynaptic vesicle release (Mallart, 1993; Kamiya and Zucker, 1994; Zucker, 1989, 1999; Dittman et al., 2000), may provide only a minor or transient contribution to enhanced vesicle release during the development and long-term strengthening of junctional signal transmission (Fig. 6). Instead, a persistent enhancement of vesicle release relies primarily on the recruitment of active zones (Fig. 7). This conclusion was further supported by our yCam2-based $\mathrm{Ca}^{2+}$ imaging results, which together with our ultrastructural data and previous observations (Lnenicka and Keshishian, 2000; Harlow et al., 2001) revealed that evoked presynaptic $\mathrm{Ca}^{2+}$ signals correlate with the density of active zones. Our data therefore suggest that enhanced vesicle release is realized by a differential regulation of active zone density in different genotypes: NMJs of dglurII $A$-ko mutants compensate for their postsynaptic defect by packing more active zones into preexisting boutons. This leads to a functional compensation, which approaches homeostasis of evoked junctional signal transmission compared with wild type (Petersen et al., 1997) presumably to ensure muscle contraction and animal survival. In contrast, enhanced junctional signal transmission as seen in elav-Campabp animals is mediated by distributing added active zones into newly grown boutons. This leads to homeostasis of active zone density compared with wild-type controls and therefore may reflect the cellular basis of strengthened junctional signal transmission at Drosophila NMJs (Fig. 9).

Previous ultrastructural observations from other Drosophila mutants and larvae of the flesh fly Sarcophaga bullata have already suggested that the density of active zones is tightly regulated (Meinertzhagen et al., 1998; Sigrist et al., 2002), presumably to ensure that individual synapses have sufficient access to, e.g., reserve pool vesicles, vesicle recycling machinery, efficient $\mathrm{Ca}^{2+}$ buffering systems, or neurotransmitter uptake mechanisms $(\mathrm{Ku}-$ romi and Kidokoro, 2002). Our data from wild-type and elavCampabp animals show a similar active zone density and evoked $\mathrm{Ca}^{2+}$ signals per bouton and thus suggest that individual boutons represent functional compartments that are likely to be maintained constant during junctional development and its strengthening. This seems to guarantee uncompromised signal transmission on a single bouton level. From these observations a model emerged that predicts that additional active zones need to be distributed in newly grown boutons (Fig. 9). This would explain the increasing number of genotypes that show a strict relationship between bouton number and transmission strength (Cheung et al., 1999; Sigrist et al., 2000, 2002; Sanyal et al., 2002). Intriguingly, in several other systems the recruitment of active synapses (Isaac et al., 1995; Liao et al., 1995; Bolshakov et al., 1997; Malenka and Nicoll, 1997; Feldman et al., 1999; Poncer and Malinow, 2001) as well as local morphological alterations of synaptic compartments (Engert and Bonhoeffer, 1999; MaleticSavatic et al., 1999) have been observed and are thought to represent long-lasting changes in the strength of synaptic communication.

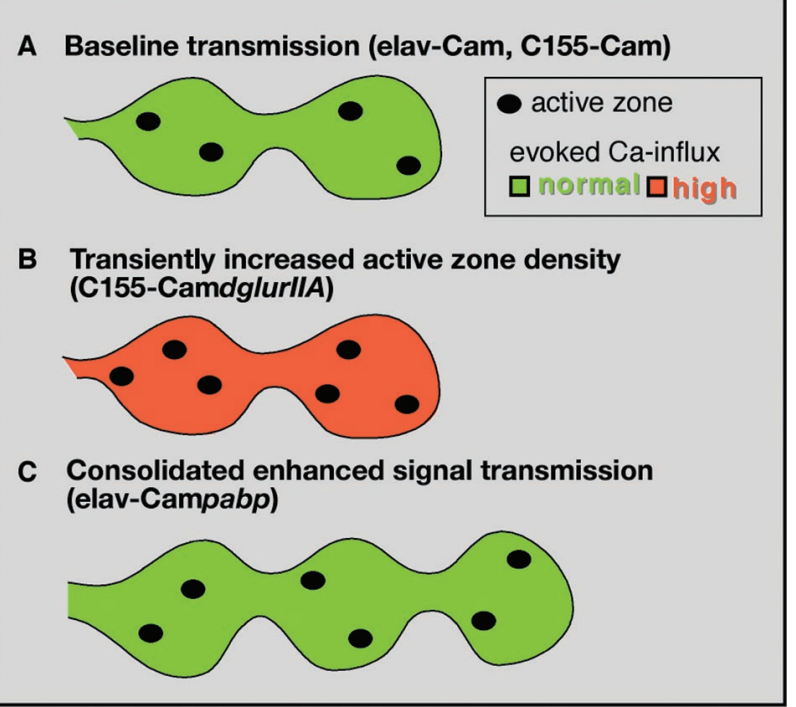

Figure 9. Potential similarities of the cellular mechanisms underlying the persistent strengthening of signal transmission during development and plasticity of Drosophila NMJs. A, Under resting conditions, junctional boutons harbor T-bar-containing active zones at a certain density (Meinertzhagen et al., 1998) (Fig. 7). Nerve activity therefore results in a typical evoked $\mathrm{Ca}^{2+}$ influx (green) into presynaptic terminals (Fig. 6) and consequently in characteristic postsynaptic responses to released neurotransmitter (Figs. 4, 8). B, Reduced muscle depolarization during muscle growth (Petersen et al., 1997; Paradis et al., 2001) and elevated subsynaptic protein synthesis during activity-dependent long-term strengthening (Sigrist et al., 2000) generate retrograde signals that result in the recruitment of active zones to enhance evoked vesicle release. This leads to a transient increase in the density of active zones per bouton (Fig. 7), which is associated with an enhanced $\mathrm{Ca}^{2+}$ influx into presynaptic terminals (red) (Fig. 6B). High active zone density and enhanced $\mathrm{Ca}^{2+}$ influx seem to compromise junctional signal transmission (as seen in dglurIIA-ko mutants) (Fig. 8). Active zone density and the associated $\mathrm{Ca}^{2+}$ dynamics are typically reset to normal by equally distributing active zones in newly grown boutons $(C)$. Such a morphological consolidation of altered junctional signal transmission ensures that each active synapse can operate normally, although the total junctional signal transmission is strengthened (as seen in elav-Campabp animals). In addition, these NMJs can now cycle through further rounds of long-term strengthening. Morphological consolidation of functional alterations therefore appears well suited for developing synaptic systems.

On the basis of the above considerations, it appears surprising that DGluR-IIA-ko mutants pack the additional active zones in a smaller number of presynaptic boutons. This results in an increased density of active zones (Fig. 7), a larger stimulationevoked $\mathrm{Ca}^{2+}$ entry per bouton (Fig. 6A), an enhanced evoked vesicle release, and a wild-type muscle depolarization on single action potentials (Fig. 4). These phenotypes show that mutants with impaired postsynaptic glutamate receptor function are capable of efficiently triggering the recruitment of active zones to compensate for its postsynaptic defect. However, it fails to induce the proportional outgrowth of new boutons that can be observed at wild-type NMJs and several other genotypes. Indeed, a recent analysis of the role of DGluR-IIA subunits in junctional development revealed that the increased expression of DGluR-IIA is sufficient to induce bouton outgrowth (Sigrist et al., 2002). Although it is currently not clear why DGluR-IIA-ko mutants accumulate active zones at such an unusual density (Fig. 7), it appears that this mechanism alone is not sufficient to ensure uncompromised repetitive signal transmission (Fig. 8). Although the latter may be attributable to increased postsynaptic desensitization in 
this mutant (Heckmann et al., 1996; Adelsberger et al., 1997; Heckmann and Dudel, 1997; DiAntonio et al., 1999), presynaptic factors like the depletion of the readily releasable vesicle pool (Dobrunz and Stevens, 1999; Kuromi and Kidokoro, 1999) also appear likely to contribute to this observation. It is therefore tempting to speculate that this mutant is trapped in a transient phase of junctional strengthening (Fig. 9).

According to such a model (Fig. 9), a postsynaptic sensor (Paradis et al., 2001) would trigger signals that control the recruitment of active zones. The transiently increased density of active zones would trigger a second signal that instructs the resetting of active zone density by distributing them into newly grown boutons. Intriguingly, the chronically hyperactive mutant eag, $S h$ represents a precedence for this scenario because it shows, presumably because of the continuous hyperactivity stimulus, an increased density of T-bar-harboring active zones (Jia et al., 1993) in an already increased number of junctional boutons (Budnik et al., 1990). These findings provide further evidence for the suggestion that developmental processes and activity-dependent phenomena may use closely related mechanisms (Goodman and Shatz, 1993; O'Leary, 1994).

\section{REFERENCES}

Adelsberger H, Heckmann M, Dudel J (1997) The amplitude of quantal currents is reduced during short-term depression at neuromuscular synapses in Drosophila. Neurosci Lett 255:5-8.

Atwood HL, Wojtowicz JM (1999) Silent synapses in neural plasticity: current evidence. Learn Mem 6:542-571.

Atwood HL, Govind CK, Wu CF (1993) Differential ultrastructure of synaptic terminals on ventral longitudinal abdominal muscles in Drosophila larvae. J Neurobiol 24:1008-1024.

Bolshakov VJ, Golan H, Kandel ER, Siegelbaum SA (1997) Recruitment of new sites of synaptic transmission during the cAMP-dependent late phase of LTP at CA3-CA1 synapses in the hippocampus. Neuron 19:635-651.

Brand AH, Perrimon N (1993) Targeted gene expression as a means of altering cell fates and generating dominant phenotypes. Development 118:401-415.

Bronk P, Wenniger JJ, Dawson-Scully K, Guo X, Hong S, Atwood HL, Zinsmaier KE (2001) Drosophila Hsc70-4 is critical for neurotransmitter exocytosis in vivo. Neuron 30:475-488.

Budnik V, Zhong Y, Wu CF (1990) Morphological plasticity of motor axon terminals in Drosophila mutants with altered excitability. J Neurosci 10:3754-3768.

Cattaert D, Birman S (2001) Blockade of the central generator of locomotor rhythm by noncompetitive NMDA receptor antagonists in Drosophila larvae. J Neurobiol 48:58-73.

Catterall WA (1998) Structure and function of neuronal $\mathrm{Ca}^{2+}$ channels and their role in neurotransmitter release. Neuron 24:307-323.

Chan FKM, Siegel RM, Zacharias D, Swofford R, Holmes KL, Tsien RY, Lenardo MJ (2001) Fluorescence resonance energy transfer analysis of cell surface receptor interactions and signaling using spectral variants of the green fluorescent protein. Cytometry 44:361-368.

Cheung US, Shayan AJ, Boulianne GL, Atwood HL (1999) Drosophila larval neuromuscular junctions responses to reduction of cAMP in the nervous system. J Neurobiol 40:1-13.

Cooper RL, Marin L, Atwood HL (1995) Synaptic differentiation of a single motor neuron: conjoint definition of transmitter release, presynaptic calcium signals, and ultrastructure. J Neurosci 15:4209-4222.

Cooper RL, Winslow JL, Govind CK, Atwood HL (1996) Synaptic structural complexity as a factor enhancing probability of calciummediated transmitter release. J Neurophysiol 75:2451-2466.

Davis GW, Goodman CS (1998) Synapse-specific control of synaptic efficacy at the terminals of a single neuron. Nature 392:82-86.

Davis GW, Schuster CM, Goodman CS (1996) Genetic dissection of structural and functional components of synaptic plasticity: III. CREB is necessary for presynaptic functional plasticity. Neuron 17:669-679.

Davis GW, DiAntonio A, Petersen SA, Goodman CS (1998) Postsynaptic PKA controls quantal size and reveals a retrograde signal that regulates presynaptic transmitter release in Drosophila. Neuron 20:305-315.

Dawson-Scully K, Bronk P, Atwood HL, Zinsmaier KE (2000) Cysteinestring protein increases the calcium sensitivity of neurotransmitter exocytosis in Drosophila. J Neurosci 20:6039-6047.

DelCastillo J, Katz B (1954) Statistical factors involved in neuromuscular facilitation and depression. J Physiol (Lond) 124:574-585.
DiAntonio A, Petersen SA, Heckmann M, Goodman CS (1999) Glutamate receptor expression regulates quantal size and quantal content at the Drosophila neuromuscular junction. J Neurosci 19:3023-3032.

Dittman JS, Kreitzer AC, Regehr WG (2000) Interplay between facilitation, depression and residual calcium at three presynaptic terminals. J Neurosci 20:1374-1385.

Dobrunz LE, Stevens CF (1999) Response of hippocampal synapses to natural stimulation patterns. Neuron 22:157-166.

Engert F, Bonhoeffer T (1999) Dendritic spine changes associated with hippocampal long-term synaptic plasticity. Nature 399:66-70.

Fan GY, Fujisaki H, Miyawaki A, Tsay RK, Tsien RY, Ellisman MH (1999) Video-rate scanning two-photon excitation fluorescence microscopy and ratio imaging with chameleons. Biophys J 76:2412-2420.

Feldman DE, Nicoll RA, Malenka RC (1999) Synaptic plasticity at thalamocortical synapses in developing rat somatosensory cortex: LTP, LTD and silent synapses. J Neurobiol 41:92-101.

Feller MB, Delaney KR, Tank DW (1996) Presynaptic calcium dynamics at the frog retinotectal synapse. J Neurophysiol 76:381-400.

Goodman CS, Shatz CJ (1993) Developmental mechanisms that generate precise patterns of neuronal connectivity. Cell 72:77-98.

Griesbeck O, Baird GS, Campbell RE, Zacharias DA, Tsien RY (2001) Reducing the environmental sensitivity of yellow fluorescent protein. J Biol Chem 276:29188-29194.

Grynkiewicz G, Poenie M, Tsien RY (1985) A new generation of Ca indicators with greatly improved fluorescence properties. J Biol Chem 260:3440-3450.

Harata N, Pyle JL, Aravanis AM, Mozhayeva M, Kavalali ET, Tsien RW (2001) Limited numbers of recycling vesicles in small CNS nerve terminals: implications for neural signaling and vesicular cycling. Trends Neurosci 24:637-643.

Harlow ML, Ress D, Stoschek A, Marshall RM, McMahan UJ (2001) The architecture of active zone material at the frog's neuromuscular junction. Nature 409:479-484.

Heckmann M, Dudel J (1997) Desensitization and resensitization kinetics of glutamate receptor channels from Drosophila larval muscle. J Biophys 72:2160-2169.

Heckmann M, Parzefall F, Dudel J (1996) Activation kinetics of glutamate receptor channels from wild-type Drosophila muscle. Eur J Physiol 432:1023-1029.

Honda A, Adams SR, Sawyer CL, Lev-Ram V, Tsien RY, Dostmann WRG (2001) Spatiotemporal dynamics of guanosine 3',5'-cyclic monophosphate revealed by a genetically encoded, fluorescent indicator. Proc Natl Acad Sci USA 98:2437-2442.

Isaac JT, Nicoll RA, Malenka RC (1995) Evidence for silent synapses: implications for the expression of LTP. Neuron 15:427-434.

Jia X-X, Gorczyca M, Budnik V (1993) Ultrastructure of neuromuscular junctions in Drosophila: comparison of wild type and mutants with increased excitability. J Neurobiol 24:1025-1044.

Kamiya H, Zucker RS (1994) Residual $\mathrm{Ca}^{2+}$ and short-term synaptic plasticity. Nature 371:602-606.

Karunanithi S, Georgiou J, Charlton MP, Atwood HL (1997) Imaging of calcium in Drosophila larval motor nerve terminals. J Neurophysiol 78:3465-3467.

Kerr R, Lev-Ram V, Baird G, Vincent P, Tsien RY, Schafer WR (2000) Optical imaging of calcium transients in neurons and pharyngeal muscles of C. elegans. Neuron 26:583-594.

Kurdyak P, Atwood HL, Stewart BA, Wu CF (1994) Differential physiology and morphology of motor axons to ventral longitudinal muscles in larval Drosophila. J Comp Neurol 350:463-472.

Kuromi H, Kidokoro Y (1999) The optically determined size of exo/ endo cycling vesicle pool correlates with the quantal content at the neuromuscular junction of Drosophila larvae. J Neurosci 19:1557-1565.

Kuromi H, Kidokoro Y (2002) Selective replenishment of two vesicle pools depends on the source of $\mathrm{Ca}^{2+}$ at the Drosophila synapse. Neuron 35:333-343.

Liao D, Hessler NA, Malinow R (1995) Activation of postsynaptically silent synapses during pairing-induced LTP in CA1 of hippocampal slice. Nature 375:400-404.

Lnenicka GA, Keshishian H (2000) Identified motor terminals in Drosophila larvae show distinct differences in morphology and physiology. J Neurobiol 43:186-197.

Malenka RC, Nicoll RA (1997) Silent synapses speak up. Neuron 19:473-476.

Maletic-Savatic M, Malinow R, Svoboda K (1999) Rapid dendritic morphogenesis in CA1 hippocampal dendrites induced by synaptic activity. Science 283:1923-1927.

Mallart A (1993) Calcium-dependent modulation of the facilitation of transmitter release at neuromuscular junctions of Drosophila. J Physiol (Lond) 87:83-88.

Meinertzhagen IA, Govind CK, Stewart BA, Carter JM, Atwood HL (1998) Regulated spacing of synapses and presynaptic active zones at larval neuromuscular junctions in different genotypes of the flies Drosophila and Sarcophaga. J Comp Neurol 393:482-492.

Miyawaki A, Llopis J, Heim R, McCaffery JM, Adams JA, Ikura M, 
Tsien RY (1997) Fluorescent indicators for $\mathrm{Ca}^{2+}$ based on green fluorescent protein and calmodulin. Nature 388:882-887.

Miyawaki A, Griesbeck O, Heim R, Tsien RY (1999) Dynamic and quantitative $\mathrm{Ca}^{2+}$ measurements using improved chameleons. Proc Natl Acad Sci USA 96:2135-2140.

Nagai T, Sawano A, Park ES, Miyawaki A (2001) Circularly permuted green fluorescent proteins engineered to sense $\mathrm{Ca}^{2+}$. Proc Natl Acad Sci USA 98:3197-3202.

O'Leary DDM (1994) Development critical period plasticity, and adult reorganizations of mammalian somatosensory systems. Curr Biol 4:535-544.

Paradis S, Sweeney ST, Davis GW (2001) Homeostatic control of presynaptic release is triggered by postsynaptic membrane depolarization. Neuron 30:737-749.

Petersen SA, Fetter RD, Noordermeer JN, Goodman CS, DiAntonio A (1997) Genetic analysis of glutamate receptors in Drosophila reveals a retrograde signal regulating presynaptic transmitter release. Neuron 19:1237-1248.

Poncer JC, Malinow R (2001) Postsynaptic conversion of silent synapses during LTP affects synaptic gain and transmission dynamics. Nat Neurosci 4:989-996.

Rosay P, Armstrong JD, Wang Z, Kaiser K (2001) Synchronized neural activity in the Drosophila memory centers and its modulation by amnesiac. Neuron 30:759-770.

Sanyal S, Sandstrom DJ, Hoeffer CA, Ramaswami M (2002) AP1 functions upstream of CREB to control synaptic plasticity in Drosophila. Nature 416:870-874.

Schuster CM, Davis GW, Fetter RD, Goodman CS (1996) Genetic dissection of structural and functional components of synaptic plasticity. II. Fasciclin II controls presynaptic structural plasticity. Neuron 17:655-667.

Siegel MS, Isacoff EY (2000) Green fluorescent protein-based sensors for detecting signal transduction and monitoring ion channel function. In: Methods in enzymology (Thorner J, Emr SD, Abelson JA, eds), pp 249-259. San Diego: Academic.

Sigrist JS, Thiel PR, Reiff DF, Lachance PED, Lasko P, Schuster CM (2000) Postsynaptic translation affects the efficacy and morphology of neuromuscular junctions. Nature 405:1062-1065.
Sigrist JS, Thiel PR, Reiff DF, Schuster CM (2002) The postsynaptic glutamate receptor subunit DGluR-IIA mediates long-term plasticity in Drosophila. J Neurosci 22:7362-7372.

Sinha SR, Wu LG, Sagau P (1997) Presynaptic calcium dynamics and transmitter release evoked by single action potentials at mammalian central synapses. Biophys J 72:637-651.

Stewart BA, Atwood HL, Renger JJ, Wang J, Wu CF (1994) Improved stability of Drosophila larval neuromuscular preparations in haemolymph-like physiological solutions. J Comp Physiol 175:179-191.

Tank DW, Regehr WG, Delany KR (1995) A quantitative analysis of presynaptic calcium dynamics that contribute to short-term enhancement. J Neurosci 15:7940-7952.

Thastrup O, Cullen PJ, Drobak BK, Hanley MR, Dowson AP (1990) Thapsigargin, a tumor promoter, discharges intracellular $\mathrm{Ca}^{2+}$ stores by specific inhibition of the endoplasmic reticulum $\mathrm{Ca}^{2+}$-ATPase. Proc Natl Acad Sci USA 87:2466-2470.

Truong K, Sawano A, Mizuno H, Hama H, Tong KI, Mal TK, Miyawaki A, Ikura M (2001) FRET-based in vivo $\mathrm{Ca}^{2+}$ imaging by a new calmodulin-GFP fusion molecule. Nat Struct Biol 8:1069-1073.

Trussell LO, Zhang S, Raman IM (1993) Desensitization of AMPA receptors upon multiquantal neurotransmitter release. Neuron 10:1185-1196.

Umbach JA, Saitoe M, Kidokoro Y, Gundersen CB (1998a) Attenuated influx of calcium ions at nerve endings of csp and shibire mutant Drosophila. J Neurosci 18:3233-3240.

Umbach JA, Grasso A, Zurcher SD, Kornblum HI, Mastrogiacomo A, Gundersen CB (1998b) Electrical and optical monitoring of alphalatrotoxin action at Drosophila neuromuscular junctions. Neuroscience 78:913-924.

Wojtowicz JM, Marin L, Atwood HL (1994) Activity-induced changes in synaptic release sites at the crayfish neuromuscular junction. J Neurosci 14:3688-3703.

Zucker RS (1989) Short-term synaptic plasticity. Annu Rev Neurosci 12:13-31.

Zucker RS (1999) Calcium- and activity-dependent synaptic plasticity. Curr Opin Neurobiol 9:305-313. 